\title{
Anticonvulsants as mood stabilisers
}

\section{Richard Porter, Nicol Ferrier \& Heather Ashton}

Recent research has highlighted the fact that a significant number of patients with bipolar affective disorder have a poor outcome (Cole et al, 1993). The most common poor outcome is the development of rapid or ultra-rapid cycling, but mixed states, prolonged depression and severe mania are also associated with poor outcome. It is estimated that approximately one-third of patients with bipolar affective disorder do not respond fully to lithium and a significant number of patients discontinue lithium (Post, 1990) which may itself cause further problems.

The discovery that anticonvulsants have a therapeutic potential in mood disorders was largely serendipitous. It was noticed that these agents improved mood in some patients with epilepsy and there were reports that, in patients with epilepsy and refractory bipolar disorder, the drugs sometimes alleviated and subsequently stabilised mood disturbances (Sussman, 1997). This led to research into the use of anticonvulsants as alternatives or supplements to lithium in the treatment of bipolar affective disorder, particularly when outcome is poor.

\section{Mechanisms of action}

Findings of abnormalities on the electroencephalogram (EEG) and temporal lobe dysfunction in bipolar disorder (Cole et al, 1993), suggests an overlapping pathophysiology with epilepsy. In addition, processes which appear to be similar to 'kindling' phenomena occur in the natural history of both bipolar and unipolar depressive disorders (Post et al, 1982). Most anticonvulsant drugs with mood-stabilising properties also inhibit the electrically-induced kindling of seizure activity in animals. However, the mechanisms of antimanic and antidepressant action and prophylactic (moodstabilising) properties may not be the same as those that control seizures in epilepsy.

The mechanisms of action of anticonvulsants in epilepsy can be classified into three main categories (Upton, 1994): (a) enhancement of inhibitory (mainly g-aminobutyric acid-mediated) processes, involving $\mathrm{Cl}^{-}$ion fluxes; (b) decrease in excitatory (particularly glutamate-mediated) processes, involving $\mathrm{Mg}^{2+}$ and $\mathrm{Ca}^{2+}$ ion fluxes; and (c) modulation of membrane cation conductance $\left(\mathrm{Na}^{+}, \mathrm{Ca}^{2+}\right.$ or $\left.\mathrm{K}^{+}\right)$by effects on membrane receptors or transport mechanisms for these ions which modulate signal transduction in the neuron system. Many anticonvulsants exert more than one of these effects.

Other modes of action may be more important in their effects in bipolar affective disorder. Post et al (1992) suggest that anticonvulsants exert an effect on second messenger systems (adenylate cyclase and phosphoinositide) which are linked to the central actions of catecholamines. These effects are also seen with lithium. In keeping with this suggestion, there is evidence of disturbance of second messenger systems and of neuronal signal transduction in both bipolar and unipolar depressive disorders (Warsh \& $\mathrm{Li}, 1996$ ). There also appear to be direct effects of anticonvulsants on monoamines. For instance, carbamazepine and valproate appear to reduce dopamine turnover (Maitre et al, 1984), and valproate was found to increase the cortisol response to L-5-HTP (Maes et al, 1997) in patients with manic disorders, suggesting an enhancement of serotonergic transmission.

Another factor in the mood-stabilising effects of anticonvulsants may be the ability to limit excessive neuronal activity, whether excitatory or inhibitory, without affecting basal activity. For example, lithium

Richard Porter is a Lecturer at the Department of Psychiatry, University of Newcastle upon Tyne (School of Neurosciences and Psychiatry, Department of Psychiatry, The Royal Victoria Infirmary, Queen Victoria Road, Newcastle upon Tyne NE1 4LP). Nicol Ferrier is Head of Department there. They have research interests in the neurobiology and treatment of affective disorders and run an academic and regional service for chronic affective disorders. Heather Ashton is Emeritus Professor of Clinical Psychopharmacology at the University of Newcastle upon Tyne, and has research interests in the same areas. 
inhibits neurotransmitter-stimulated activation of adenylate cyclase, but has little effect on basal activity. Post et al (1992) suggest that carbamazepine and other anticonvulsants may also have these effects. Similarly, gabapentin reduces abnormal pain sensitivity owing to increased excitability of dorsal horn neurones, but has no effect on physiological pain where there is no pre-existing hyper-excitability (Field et al, 1997).

\section{Forced normalisation}

Trimble (1996) has reviewed the phenomenon of 'forced normalisation', a term describing the apparently reciprocal relationship between psychosis and epileptic seizures. It had previously been noted in patients with epilepsy (usually with severe and previously intractable epilepsy) that control of seizures and normalisation of the EEG with an anticonvulsant drug sometimes precipitated psychosis or an affective disorder. Such precipitation, also termed 'alternative psychosis', was observed with succinimides, phenacetin, barbiturates, hydantoins and carbamazepine and has recently been reported with the newer anticonvulsants. Withdrawal of anticonvulsants in patients with epilepsy who have developed psychosis as a result of forced normalisation results in reappearance of the seizures and resolution of the abnormal mental state. However, some anticonvulsants can cause mood disorders or psychosis independently of seizure control, the significance of which in the use of anticonvulsants as mood stabilisers in patients with affective disorders is unclear.

\section{Individual drugs}

\section{Carbamazepine}

Carbamazepine is a dibenzazepine derivative. Sideeffects include reversible and dose-related blurred vision, dizziness, ataxia and gastro-intestinal disturbance. Therapy should be initiated at low doses and gradually increased. Use of a slow-release preparation, which can be given twice daily, is advantageous, particularly when compliance is likely to be a problem. We suggest a starting dose of $400 \mathrm{mg}$ once a day, increased by $200-400 \mathrm{mg}$ at a time as indicated by side-effects and clinical response. There is no evidence of a direct relationship between blood levels and response in affective disorders. The dose should therefore be titrated to maximise clinical effectiveness while minimising side-effects, in each individual. Doses of around $800 \mathrm{mg}$ per day seem to be effective.
Rashes are common and should be monitored closely since severe reactions can occur. Treatment should be withdrawn if the rash worsens or is accompanied by other symptoms. Blood disorders can occur and a full blood count should be performed before and at intervals during therapy. Carbamazepine is metabolised in the liver by the cytochrome P450 3A4 isoform and also induces this isoform. An important consequence of this is a reduction in blood levels of oral contraceptives and either higher doses of oral contraceptives or alternative methods of birth control should be used. Since some selective serotonin reuptake inhibitors and nefazadone may inhibit the metabolism of carbamazepine, care should taken if these or any other drug known to inhibit cytochrome P450 3A4 is co-prescribed (see Nemeroff et al, 1996 for review).

\section{Prophylaxis}

Evidence suggests that carbamazepine has a prophylactic effect in bipolar affective disorder (see Post et al, 1996 for review). It appears to be equally effective in reducing the frequency of manic and depressive episodes. However, a recent randomised multi-centre study (Greil et al, 1997) in 144 patients over 2.5 years, found that lithium was superior to carbamazepine in terms of recurrence rate, discontinuation owing to adverse effects, and the need for co-medication. Patients on carbamazepine reported fewer side-effects. Therefore, although carbamazepine monotherapy does appear to have a beneficial effect in prophylaxis, it is probably best reserved as an alternative when lithium is contraindicated or not tolerated. There is also evidence that in monotherapy, like lithium, it may lose effectiveness over time (Post et al, 1990).

Addition of carbamazepine to lithium may be useful in treatment-resistant or partially treatmentresistant patients. Denicoff et al (1994) carried out a study in which patients received monotherapy for one year with lithium or carbamazepine, followed by a cross-over year, followed by a year on combination therapy. They found that the combination was more effective than either monotherapy. A retrospective study of 18 patients also suggested that those on combination therapy with carbamazepine and lithium may function better (Kishimoto, 1992) and with lower levels of lithium. These studies suggest that augmentation of lithium with carbamazepine may improve outcome. Neurotoxicity with this combination has been reported, particularly in patients with pre-existing neurological damage (Shukla et $a l, 1984)$. Generally the combination of lithium and carbamazepine appears safe, particularly if the additional drug is introduced gradually. We suggest that the addition of carbamazepine be considered early if response to lithium appears to be inadequate. 


\section{Mania}

Both carbamazepine and oxcarbazine (not available in the UK) have been studied in acute mania in various experimental designs, although no largescale placebo-controlled trials have been conducted. In a review of these studies (Post et al, 1996) it was concluded that 123 out of 203 patients had a markedto-excellent antimanic response. Comparisons have been made both with standard neuroleptics and with lithium. Generally, double-blind comparisons with neuroleptics (chlorpromazine or haloperidol) have found equivalent efficacy, but there is a suggestion that neuroleptics give rise to a greater incidence of side-effects. Two studies have reported an advantage of neuroleptics over carbamazepine in the first few days of treatment (Brown et al, 1989; Emrich, 1990). Studies comparing carbamazepine with lithium show no consistent difference between the two agents. Combination therapy may be appropriate in acute mania. Small et al (1996), in a series of studies, investigated the effects of carbamazepine plus lithium against haloperidol plus lithium and electroconvulsive therapy plus lithium in the treatment of acute mania. In double-blind placebo-controlled trials, the three combinations were equally effective. There were fewer neurological side-effects on the combination of lithium plus carbamazepine.

\section{Depression}

A number of open and small-scale controlled studies of carbamazepine in depression suggest a possible antidepressant response, but do not constitute clear evidence.

\section{Valproate}

Valproate is a simple branched-chain fatty acid. It has good efficacy in epilepsy and is generally well tolerated. It is contraindicated in active liver disease or where there is a family history of hepatic dysfunction. Liver function should be monitored at baseline and regularly thereafter, particularly during the first six months of treatment, since impaired hepatic function may occur, leading rarely to fatal hepatic failure. Treatment should be withdrawn if vomiting, anorexia, jaundice or drowsiness occur. The most common side-effects are gastric irritation, nausea, ataxia and tremor. Increased appetite and weight gain can be a problem, particularly in combination with other drugs that have these effects. Hair loss is a rare but distressing side-effect. The slow-release preparation may be given once daily.

Metabolism is complicated and occurs via a variety of conjugation and oxidative pathways. The plasma elimination half-life is shortened by the coadministration of hepatic enzyme inducers such as carbamazepine. Valproate does not, however, induce its own metabolism or that of other drugs such as oral contraceptives. Most patients will tolerate a starting dose of $400 \mathrm{mg}$ twice a day which can then be gradually titrated upwards.

No studies have compared carbamazepine with valproate directly and apart from a few specific situations (see 'Clinical predictors of response'), the decision regarding which of these alternatives to use depends largely on side-effect profile and the presence of other drugs with which interactions may occur.

\section{Prophylaxis}

Evidence regarding prophylaxis is less good for valproate than for carbamazepine, and large-scale double-blind comparisons with other agents have not yet been reported (except in conference presentations which suggest a prophylactic effect). Open studies of valproate as monotherapy suggest that valproate is effective in prophylaxis of mania, but that its effectiveness in preventing depressive episodes is limited (Post et al, 1996). In combination, a small-scale double-blind study of lithium plus valproate compared with lithium plus placebo (Solomon et al, 1997) suggested a significant advantage of the combination over monotherapy. There are also case report series in rapid-cycling patients suggesting that the combination of valproate plus lithium is effective. The potential benefits of any combination must be balanced against the risks of side-effects or interactions. However, Granneman et al (1996) studied the combination of valproate plus lithium in 16 healthy volunteers and, although they found a small increase in valproate levels, there was no increase in adverse events.

\section{Mania}

Several controlled trials suggest an acute antimanic effect of valproate. The largest randomised, doubleblind controlled trial compared valproate with lithium and with placebo in 179 patients with acute mania (Bowden et al, 1994). Similar efficacy was found for lithium and valproate with both being significantly more effective than placebo. Further analysis of the data (Swann et al, 1997) suggests a particularly poor response to lithium and a good response to valproate in the presence of depressive symptoms. This study demonstrated only a $50 \%$ response rate with either monotherapy and once again combination therapies may be the best option. The effects of both lithium plus valproate and valproate plus neuroleptics in mania need further evaluation.

Schaff et al (1993) reported experience with 29 patients in whom valproate was added to carbam- 
azepine therapy and 35 in whom valproate was added to a combination of carbamazepine and lithium. There were good response rates in both mania and rapid cycling following this addition and a low incidence of side-effects. Such a combination of three mood stabilisers may be necessary and useful in refractory cases. Blood levels should be monitored since large doses of valproate may be needed because of enzyme induction by carbamazepine. Valproate may inhibit the metabolism of carbamazepine and displace it from protein binding. Neurotoxicity has occasionally been reported with this combination even with relatively low levels of valproate (Onady \& Calabrese, 1989).

\section{Depression}

Only open studies suggest a response in a relatively small proportion of patients, which may be little more than placebo.

\section{Monitoring}

Little is known regarding the relationship between blood levels of valproate and effectiveness in depression or prophylaxis. A study in 65 hospitalised patients with acute mania showed that response was more likely if levels were above $45 \mathrm{mg} / \mathrm{l}$, whereas above $125 \mathrm{mg} / \mathrm{l}$ adverse events were more likely (Bowden et al, 1996). Other studies suggest that a level above $100 \mathrm{mg} / \mathrm{l}$ gives rise to increased risk of cognitive impairment and side-effects. We would, therefore, suggest that for acute mania a level of around $60-100 \mathrm{mg} / 1$ is optimal. In prophylaxis there is no direct evidence and the dose should be titrated according to clinical response. However, response cannot be predicted until a relapse has occurred. We suggest that the dose should be increased until side-effects are experienced or the level is in the range $60-100 \mathrm{mg} / \mathrm{l}$.

\section{Gabapentin}

Gabapentin is an antiepileptic agent structurally related to $\gamma$-aminotbutyric acid (GABA). It is used as an add-on therapy in patients with partial seizures resistant to conventional therapies. Gabapentin increases GABA release, increases the breakdown of glutamic acid and binds to a novel site on voltagedependent calcium channels (see Brown et al, 1996 for review). It also reduces the potassium-induced release of several monoamine neurotransmitters (noradrenaline, dopamine, serotonin) from brain tissue in vitro (Reimann et al, 1983).

Gabapentin is not metabolised in humans. It is not protein bound, has no known pharmacokinetic interactions with valproate, carbamazepine, other anticonvulsants or oral contraceptives and is renally excreted unchanged. Gabapentin has a high therapeutic index and a relatively benign side-effect profile. Side-effects reported with gabapentin are transient and minor, the most common being somnolence, dizziness, ataxia and fatigue. It is not associated with haematological or hepatic problems and does not require serum concentration monitoring. These properties make gabapentin an attractive medication choice for patients with bipolar affective disorder who are receiving multiple psychotropics, those with hepatic or haematological disturbance and those in whom blood monitoring of levels is problematic. In addition to these pharmacokinetic and pharmacological advantages there are theoretical reasons why gabapentin may be of interest in mood disorders.

\section{Mood effects in epilepsy}

There have been a variety of open studies of patients with epilepsy which have reported positive benefits of gabapentin on mood, cognition and quality of life. Dimond et al (1996) reviewed five double-blind clinical trials of gabapentin as an add-on therapy in 700 patients with epilepsy. Of the patients treated with gabapentin $46 \%$ reported improvements in their general well-being compared with $29 \%$ of the placebo-treated patients. Caution is required since this paper is based on a retrospective examination of data from trials in epilepsy where psychological effects were not the primary focus and there was often inadequate use of validated mood ratings. However, it appears that the beneficial effects of gabapentin on mental function in add-on therapy trials differs from the usual reports of the negative influence of antiepileptic polytherapy on attention, concentration, drive, mood and sociability. It is noteworthy that some patients reported improvement in mood even in the absence of seizure control.

\section{Box 1. Treatment of acute mania}

Carbamazepine, valproate and lithium are probably equipotent, but all have limitations as monotherapy

Lithium is relatively ineffective in mania with co-existing depressive symptoms

Combinations may be more effective research is needed on combinations of a mood stabiliser plus neuroleptics and/or two mood stabilisers, e.g. carbamazepine plus lithium 
Some case reports have described the development of disinhibition or hypomanic symptoms in patients with epilepsy (e.g. McElroy et al, 1997).

\section{Affective disorders}

No placebo-controlled or randomised studies of gabapentin in affective disorder have been reported. Two case reports (Ryback \& Ryback, 1995; Stanton et al, 1997) described apparent benefits of gabapentin in patients with behavioural discontrol and refractory mania, respectively. The results of five open-label studies with gabapentin as either sole or add-on therapy suggest a good response in hypomania, bipolar depression and in the prophylaxis of bipolar disorder (summarised by Ferrier, 1998). In particular, the largest of these studies (Ryback et al, 1997) showed a positive response in 67 out of 73 patients with mixed bipolar disorder and, in particular, in rapid cycling at doses of between 1500 and $2400 \mathrm{mg}$. At this dose it is well tolerated, although care is needed to monitor for sideeffects, particularly worsening of the psychiatric state. We suggest that gabapentin be reserved as addon therapy in cases which are resistant to lithium, carbamazepine and valproate or as an alternative when these agents are not tolerated.

\section{Lamotrigine}

Lamotrigine stabilises neuronal membranes and reduces the release of excitatory amino acids, particularly glutamate, by blocking voltage-dependent sodium channels. There is also evidence that it acts as a calcium channel antagonist (Leach et al, 1986).

Lamotrigine was marketed in the UK in 1991 as an add-on treatment for patients with refractory epilepsy. The most common adverse effects are headache, nausea, diploplia, dizziness, ataxia and occasionally sedation. A skin rash, usually maculopapular, occurs in around $5 \%$ of patients early in treatment. It is more common in children, with polypharmacy (particularly with valproate, which inhibits the metabolism of lamotrigine) and where there has been a rapid escalation in dose. In most cases the rash is mild and subsides spontaneously without or with drug withdrawal, but in some patients there is an associated generalised illness with a few developing erythma multiforme, StevensJohnson syndrome and angio-oedema. There have been fatalities reported. It is therefore advisable to titrate the drug slowly and to withdraw it at the first signs of a rash. Lamotrigine does not induce P450 enzymes and has no significant pharmacokinetic effects on the concentrations of commonly used psychotropic medication. However, carbamazepine will decrease lamotrigine levels whereas valproate impairs the elimination of lamotrigine, thereby increasing the levels. Lamotrigine may also have a pharmacodynamic interaction with carbamazepine, leading to neurotoxicity (Dubovsky \& Buzan, 1997).

\section{Psychiatric effects}

Lamotrigine is of particular interest in psychiatry since its mechanism of action is significantly different from other anticonvulsants and also from other psychotropic and mood-stabilising agents. In animal studies lamotrigine does not prevent kindling, but it increases the stimulus intensity necessary to produce kindling. Lamotrigine inhibits ischaemia-induced release of the excitotoxin glutamate (Messenheimer, 1995) and it reduces neuronal damage and is associated with improved recovery after cerebral ischaemia in animals (Shuaib et al, 1995). It may, therefore, be of particular interest in patients with severe manic-depressive disorders, a significant number of whom have patchy white matter lesions (Videbech, 1997). In the normal elderly population such lesions are associated with ischaemia, but it is unclear whether this is the mechanism leading to white matter change in manic-depressive illness. Patients with cerebrovascular disease may present with secondary mania, and clinical observation indicates that ischaemic cerebrovascular disease worsens the outcome of bipolar disorder. A number of studies have reported that patients with poor outcome or bipolar disorder (with either rapid cycling or mixed mania) had an increased frequency of temporal lobe slow wave activity on the EEG (Cole et al, 1993). Thus, lamotrigine's mechanism of action indicates a good case for examining its role in psychiatric and neuropsychiatric disorder, but randomised placebocontrolled trials are awaited.

\section{Effects in patients with epilepsy}

A positive effect of lamotrigine on mood is suggested by reports that it improves global impressions of well-being in patients with epilepsy more than it improves seizure control and that patients in continuation studies elect to keep taking this medication more frequently than would be expected on the basis of improved seizure control alone (Messenheimer, 1995).

\section{Effects in bipolar disorder}

No randomised double-blind placebo-controlled studies have been published, but two have been presented at conferences. There are preliminary suggestions that lamotrigine is effective in bipolar depression, whereas most of the anticonvulsants are more effective in reducing the frequency and/or 
severity of hypomania. Case reports indicating efficacy in bipolar disorder, particularly in rapid cycling and bipolar depression have been published by a number of authors and a variety of retrospective and naturalistic studies where lamotrigine has been used as add-on or monotherapy in bipolar disorder generally show positive results. Alongside these generally positive reports of lamotrigine there have been some adverse effects including delirium, confusion, depression and psychosis (see Ferrier, 1998 for review). We suggest that, as for gabapentin, lamotrigine be reserved for resistant cases or where more conventional mood stabilisers are not tolerated.

\section{Clonazepam and lorazepam}

Benzodiazepines combine with specific sites on the GABA post-synaptic receptor complex, enhancing the effect of synaptically released GABA and increasing the frequency of opening of chloride channels. They appear to be less efficacious than other anticonvulsants as mood stabilisers (Post $e t$ $a l, 1992)$. However, clonazepam and lorazepam have been used successfully in acute mania and it is likely that these drugs may be an appropriate substitute for neuroleptics in this condition (Post et al, 1996). It is common practise to use them for rapid tranquillisation in severe mania. This reduces the dose of neuroleptics needed. Alprazolam may be less useful as it has been associated with switches to mania in patients with bipolar illness and in anxiety disorders (Post et al, 1996).

Box 2. Prophylaxis

Effectiveness of prophylaxis can in many cases be improved by the addition of an anticonvulsant

Rapid-cycling and mixed affective states predict a poor response to lithium and add-on therapy will almost certainly be necessary

Carbamazepine or valproate are appropriate add-on therapies

If lithium is not tolerated, carbamazepine or valproate may be used as monotherapy; consideration should be given to use of a combination of both

Preliminary evidence suggests that gabapentin and lamotrigine are useful as add-on agents in refractory cases

\section{Box 3. Gabapentin and lamotrigine}

Relative lack of side-effects

Can be used in addition to lithium and other anticonvulsants

Probably particularly useful in rapid-cycling, mixed affective states and when EEG abnormalities are present

Trials as add-on and monotherapy in the various stages of bipolar disorder are awaited

\section{Vigabatrin}

Vigabatrin is a synthetic derivative of GABA which potently inhibits GABA catabolism. Its use in affective disorders is virtually unexplored and may be limited by emergent depression and psychosis, which has been noted in a small number of patients with epilepsy especially in those with pre-existing, or a history of, psychiatric illness (Ring et al, 1992).

\section{Clinical predictors of response}

\section{Mixed affective states}

There is consistent evidence that a mixed state with co-existing manic and depressive syndromes predicts a poor response to lithium (Prien et al, 1988). Evidence for carbamazepine is unclear, but studies suggest a relatively good response to valproate in mixed states. In a double-blind study of lithium, valproate and placebo in 179 hospitalised patients with acute manic episodes, Swann et al (1997) found that even a modest level of pre-treatment depressive symptoms predicted a poor antimanic response to lithium and a better response to valproate. There is also some evidence that mixed affective states may predict a better prophylactic response to valproate (Calabrese et al, 1993).

\section{Rapid cycling}

Studies suggest that patients with a rapid-cycling pattern have a poorer prophylactic response to both lithium and carbamazepine than patients without such a pattern. The response to carbamazepine in rapid cycling appears to be better than the response to lithium (Okuma, 1993; Denicoff et al, 1994). This 
may be a reason for moving immediately to a combination of lithium and carbamazepine. There is evidence that the prophylactic effect of valproate may also be relatively good in this group (Calabrese et al, 1993). The study by Bowden et al (1994) suggests that the acute antimanic response to valproate is good in patients with rapid cycling.

\section{Organic factors}

Organic factors such as ischaemic lesions may predict poor outcome in bipolar disorder. Clinical experience suggests that such cases may respond relatively better to anticonvulsants than to mood stabilisers. This ties in with studies which suggest that a positive family history of affective illness in a first-degree relative is associated with a good response to lithium (Maj et al, 1984), whereas a negative family history is associated with a good response to carbamazepine (Joffe, 1991). In patients with a history of organic lesions or imaging suggestive of this, we would recommend that consideration be given to adding anticonvulsants to the treatment regime at an early stage.

\section{Conclusion}

Of the anticonvulsants discussed here only carbamazepine is currently licensed for the treatment of bipolar affective disorder in the UK. However, sodium valproate is also widely used and the newer agents show considerable promise in this area. Clinical trials have not confirmed anticonvulsants as routine alternatives to lithium in prophylaxis or the treatment of mania, but in certain clinical situations or when lithium is contraindicated or not tolerated, anticonvulsants may be a more appropriate choice. Combinations of lithium with anticonvulsants and those of two or more anticonvulsants increase the level of side-effects and the risks of toxicity. However, if such combinations are instituted carefully and side-effects are closely monitored, the risks can be minimised and such combinations are often beneficial in treatment-resistant cases. In many cases prolonged use is required before benefits are evident. Cases who are likely to respond to anticonvulsants may be predicted from the clinical history and anticonvulsants added at an early stage. The mechanisms of action of anticonvulsant drugs in bipolar disorder are still unknown although there are several candidate actions. Future research may lead to a more rational approach to combination therapy.

\section{References}

Bowden, C. L., Brugger, A. M., Swann, A. C., et al (1994) Efficacy of divalproex vs lithium and placebo in the treatment of mania. Journal of the American Medical Association, 271, 918-924.

- Janicak, P. G., Orsulak, P., et al (1996) Relation of serum valproate concentration to response in mania. American Journal of Psychiatry, 153, 765-770.

Brown, D., Silverstone, T. \& Cookson, J., (1989) Carbamazepine compared to haloperidol in acute mania. International Clinical Psychopharmacology, 4, 229-238.

-, Boden, P., Singh, L., et al (1996) Mechanisms of action of gabapentin. Reviews in Contemporary Pharmacotherapy, 7, 203-214.

Calabrese, J. R., Woyshville, M. J., Kimmel, S. E., et al (1993) Predictors of valproate response in bipolar rapid cycling. Journal of Clinical Psychopharmacology, 13, 280-283.

Cole, A. J., Scott, J., Ferrier, I. N., et al (1993) Patterns of treatment resistance in bipolar affective disorder. Acta Psychiatrica Scandinavica, 88, 121-123.

Denicoff, K. D., Blake, K. D., Smith-Jackson, E. E., et al (1994) Morbidity in treated bipolar disorders: one-year prospective study using daily life chart ratings. Depression, 2, 95-104.

Dimond, K. R., Pande, A. C., Lamoreaux, L., et al (1996) Effect of gabapentin (Neurotin) on mood and well-being in patients with epilepsy. Progress in Neuro-Psychopharmacology and Biological Psychiatry, 20, 407-417.

Dubovsky, S. L. \& Buzan, R. D. (1997) Novel alternatives and supplements to lithium and anticonvulsants for bipolar affective disorder. Journal of Clinical Psychiatry, 58, 224-242.

Emrich, H. M. (1990) Studies with oxcarbazepine (Trileptal) in acute mania. International Clinical Psychopharmacology, 5, 83-88.

Ferrier, I. N. (1998) Lamotrigine and gabapentin - alternatives in the treatment of bipolar disorder. Neuropsychobiology, 38, 192-197.

Field, M. J., Oles, R. J., Lewis, A. S., et al (1997) Gabapentin (neurontin) and S-(+)-3-isobutylgaba represent a novel class of selective antihyperalgesic agents. British Journal of Pharmacology, 121, 1513-1522.

Granneman, G. R., Schneck, D. W., Cavanaugh, J. H., et al (1996) Pharmacokentic interactions and side-effects resulting from concomitant administration of lithium and divalproex sodium. Journal of Clinical Psychiatry, 57, 204-206.

Greil, W., Ludwig-Mayerhofer, W., Erazo, N., et al (1997) Lithium versus carbamazepine in the maintenance treatment of bipolar disorders - a randomised study. Journal of Affective Disorders, 43, 151-161.

Joffe, R. T. (1991) Carbamazepine, lithium, and life course of bipolar affective disorder (letter). American Journal of Psychiatry, 148, 1270-1271.

Kishimoto, A. (1992) The treatment of affective disorder with carbamazepine: prophylactic synergism of lithium and carbamazepine combination. Progress in NeuroPsychopharmacology and Biological Psychiatry, 16, 483-493.

Leach, M. J., Marsden, C. M., \& Miller, A. A. (1986) Pharmacological studies on lamotrigine, a novel potential antiepileptic drug: II. Neurochemical studies on the mechanism of action. Epilepsia, 27, 490-497.

Maitre, L., Baltzer, V. \& Mondadori, C. (1984) Psychopharmacological and behavioural effects of antiepileptic drugs in animals. In Anticonvulsants in Affective Disorders (eds $\mathrm{H}$. M. Emrich, T. Okuma \& A. A. Muller), pp. 3-13. Amsterdam: Elsevier Science.

Maes, M., Calabrese, J., Jayathilake, K., et al (1997) Effects of subchronic treatment with valproate on L-5-HTPinduced cortisol responses in mania: evidence for increased central serotonergic neurotransmission. Psychiatry Research, 71, 67-76. 
Maj, M., Del Vecchio, M., Staace, F., et al (1984) Prediction of affective psychoses response to lithium prophylaxis. Acta Psychiatrica Scandanavia, 69, 37-44.

McElroy, S. L., Soutullo, C. A., Keck, P. E., et al (1997) A pilot trial of adjunctive gabapentin in the treatment of bipolar disorder. Annals of Clinical Psychiatry, 9, 99-104.

Messenheimer, J. A. (1995) Lamotrigine. Epilepsia, 36, S87S94.

Nemeroff, C., DeVane, C. \& Pollock, B. (1996) Newer antidepressants and the cytochrome $\mathrm{P} 450$ system. American Journal of Psychiatry, 153, 311-320.

Okuma, T. (1993) Effects of carbamazepine and lithium on affective disorders. Neuropsychobiology, 27, 138-145.

Onady, A. A. \& Calabrese, J. R. (1989) Carbamazepine autoand hetero-induction complicating clinical care. Journal of Clinical Psychopharmacology, 9, 387-388.

Post, R. M. (1990) Non-lithium treatment for bipolar disorder. Journal of Clinical Psychiatry, 51, 9-16.

-, Uhde, T. W., Putnam, F. W., et al (1982) Kindling and carbamazepine in affective illness. Journal of Nervous and Mental Disease, 170, 717-731.

-, Leverich G. S., Rosoff A. S., et al (1990) Carbamazepine prophylaxis in refractory affactive disorders: a focus on long-term follow-up. Journal of Clinical Psychopharmacology, 10, 318-323.

-, Weiss, S. R. B., Chuang, D.-M. (1992) Mechanisms of action of anticonvulsants in affective disorders: comparison with lithium. Journal of Clinical Psychopharmacology, 12, 23S-35S.

-, Ketter, T. A., Denicoff, K., et al (1996) The place of anticonvulsant therapy in bipolar illness. Psychopharmacology, 128, 115-129.

Prien, R. F., Himmelhoch, J. M. \& Kupfer, D. J. (1988) Treatment of mixed mania. Journal of Affective Disorders, 15, 9-15.

Reimann, W. (1983) Inhibition by GABA, baclofen and gabapentine of dopamine release from rabbit caudate nucleus: Are there common or different sites of action? European Journal of Pharmacology, 94, 341-344.

Ring, H. A., Trimble, M. R., Costa, D. C., et al (1992) Effect of vigabatrin on striatal dopamine receptors: evidence in humans for interactions of GABA and dopamine systems. Journal of Neurology, Neurosurgery and Psychiatry, 55, 758761.

Ryback, R. \& Ryback, L. (1995) Gabapentin for behavioural dyscontrol. American Journal of Psychiatry, 152, 1399.

-, Brodsky, L. \& Manufasi, F. (1997) Gabapentin in bipolar disorder (letter). Journal of Neuropsychiatry, 9, 301.

Schaff, M., Fawcett, J. \& Zajecka, J. (1993) Divalproex sodium in the treatment of refractory affective disorders. Journal of Clinical Psychiatry, 54, 380-384.

Shuaib, A., Mahmood, R. H. \& Wishart, T. (1995) Neuroprotective effects of lamotrigine in global ischemia in gerbils: a historical, in vivo micro-dialysis and behavioural study. Brain Research, 702, 199-206.

Shukla, S., Godwin, C. D., Long, L. E. B., et al (1984) Lithiumcarbamazepine neurotoxicity and risk factors. American Journal of Psychiatry, 141, 1604-1606.

Small, J. G., Klapper, M. H., Milstein, V., et al (1996) Comparison of therapeutic modalities for mania. Psychopharmacology Bulletin, 32, 623-627.

Solomon, D. A., Ryan, C. E., Keitner, G. I., et al (1997) A pilot study of lithium carbonate plus divalproex sodium for the continuation and maintenance treatment of patients with bipolar I disorder. Journal of Clinical Psychiatry, 58, 95-99.

Stanton, S. P., Keck, P. E. \& McElroy, S. L. (1997) Treatment of acute mania with gabapentin (letter). American Journal of Psychiatry, 154, 287.

Sussman, N. (1997) Gabapentin and lamotrigine: alternative agents for the treatment of bipolar disorder. Primary Psychiatry, August, 25-42.

Swann, A. C., Bowden, C. L., Morris, D., et al (1997) Depression during mania. Treatment response to lithium or divalproex. Archives of General Psychiatry, 54, 37-42.
Trimble, M. R. (1996) Anticonvulsant-induced psychiatric disorders. The role of forced normalisation. Drug Safety, 15, 159-166.

Upton, N. (1994) Mechanisms of action of new antiepileptic drugs: rational design and serendipitous findings. Trends in Pharmacological Sciences, 15, 456-463.

Warsh, J. J. \& Li, P. P. (1996) Second messenger systems and mood disorders. Current Opinion in Psychiatry, 9, 23-29.

Videbech, P. (1997) MRI findings in patients with affective disorder: a meta-analysis. Acta Psychiatrica Scandinavica, 96, 157-168.

\section{Multiple choice questions}

1. Known predictors of a poor response to lithium in acute mania include:

a depressive symptoms

b age over 50

c rapid cycling

d psoriasis.

2. Anticonvulsants are known to:

a reduce cortisol levels in depression

b enhance GABA-mediated processes

c modulate membrane cation conductance

d affect second messenger systems.

3. Carbamazepine:

a increases blood levels of oral contraceptives

b may cause serious blood dyscrasias

c in prophylaxis becomes more effective over time

d can safely be combined with lithium.

4. Valproate:

a may cause hepatic failure

b is ineffective in treating mania with depressive symptoms

c is less effective in prophylaxis of depressive episodes

d is metabolised more quickly when used in combination with carbamazepine.

MCQ answers

$\begin{array}{llllllll}\text { 1 } & & \text { 2 } & & \text { 3 } & & \text { 4 } & \\ \text { a } & \text { T } & \text { a } & \text { F } & \text { a } & \text { F } & \text { a } & \text { T } \\ \text { b } & \text { F } & \text { b } & \text { T } & \text { b } & \text { T } & \text { b } & \text { F } \\ \text { c } & \text { T } & \text { c } & \text { T } & \text { c } & \text { F } & \text { c } & \text { T } \\ \text { d } & \text { F } & \text { d } & \text { T } & \text { d } & \text { T } & \text { d } & \text { T }\end{array}$

